\title{
The Role of Redundant Bases and Shrinkage Functions in Image Denoising
}

\author{
Yacov Hel-Or ${ }^{1}$ and Gil Ben-Artzi ${ }^{2}$ \\ ${ }^{1}$ Efi Arazi School of Computer Science, The Interdisciplinary Center, Herzliya, Israel 30900 \\ 2 Department of Computer Science, Ariel University, Ariel, Israel 40700
}

\begin{abstract}
Wavelet denoising is a classical and effective approach for reducing noise in images and signals. Suggested in 1994 [1], this approach is carried out by rectifying the coefficients of a noisy image in the transform domain, using a set of scalar shrinkage function (SFs). A plethora of papers deals with the optimal shape of the SFs and the transform used, where it is known that applying the SFs in redundant bases provides improved results. This paper provides a complete picture of the interrelations between the transform used, the optimal shrinkage functions, and the domains in which they are optimized. In particular, we show that for subband optimization, where each SF is optimized independently for a particular band, optimizing the SFs in the spatial domain is always better than or equal to optimizing the SFs in the transform domain. For redundant bases, we provide the expected denoising gain we may achieve, relative to the unitary basis, as a function of the redundancy rate.
\end{abstract}

Index Terms-Wavelet transforms, image restoration, image denoising, shrinkage denoising, cycle spinning, noise removal, overcomplete representation.

\section{INTRODUCTION}

Consider a noisy image

$$
\mathbf{y}=\mathbf{x}+\mathbf{n}
$$

where $\mathbf{y}$ is the observed image, $\mathbf{x}$ the unknown original image and $\mathbf{n}$ the contaminating noise (all in vector notation). The goal is to reconstruct the original image $\mathbf{x}$ given the noisy measurement $\mathbf{y}$. This is a classical formulation of image denoising, which is a typical instance of an inverse problem. Using the maximum a posteriori (MAP) criterion, the solution aims at maximizing the a posteriori probability given the noisy image. The MAP solution must consider prior knowledge about the distribution of $\mathbf{x}$, and generally speaking, the prior distribution of natural images or any other specific class of images plays a key role in any denoising approach.

In the last few years, with the emergence of deep neural networks (DNN), a large body of works suggests performing image denoising by feedforward neural networks when the network aims at learning image-specific or general statistics of natural images [2]-[5]. Although DNN approaches are very effective and are the main focus these days, in this paper we remain loyal to the classical approaches where denoising is applied in the transform domain using shrinkage mappings. The reason for taking this position is that we are motivated by the theoretical bounds and the insights we gain by analyzing these type of approaches.

Transform based denoising is often implemented using some type of wavelet transform. The main motivation for this approach stems from the observation that the wavelet transform of natural images tends to reduce pixel dependencies [6]-[9]. Hence, it is possible to make a reasonable estimate about the joint distribution of the wavelet coefficients from their marginal distributions. When dealing with image denoising, this leads to a family of classical techniques known as the

Corresponding author: Y. Hel-Or (email: toky@idc.ac.il). wavelet shrinkage methods, first introduced by Donoho and Johnstone in $1994[1],[10],[11]$. The shrinkage denoising approach is composed of a wavelet transform:

$$
\mathbf{y}_{u}=U \mathbf{y}
$$

where $U$ is a matrix comprising the transform basis. The transform coefficients are then rectified by a correction step in which they are modified according to a set of scalar shrinkage functions, $\left\{\psi_{i}: \Re \rightarrow \Re\right\}$ :

$$
\tilde{\mathbf{y}}_{u}=\psi\left(\mathbf{y}_{u}\right)
$$

where $\psi=\left(\psi_{1}, \psi_{2}, \cdots\right)$ is a vector of scalar mapping functions applied to each coefficient independently: $\tilde{\mathbf{y}}_{u}[i]=$ $\psi_{i}\left(\mathbf{y}_{u}[i]\right)$. The denoised image is then obtained by applying the pseudo-inverse transform to the modified coefficients:

$$
\tilde{\mathbf{y}}_{u}^{\mathcal{S}}=U^{+} \tilde{\mathbf{y}}_{u}
$$

where the superscript $\mathcal{S}$ indicates that we have transformed back to the spatial (image) domain. In cases where the transform is unitary or a tight frame, the pseudo-inverse yields the adjoint; thus, $\tilde{\mathbf{y}}_{u}^{\mathcal{S}}=U^{T} \tilde{\mathbf{y}}_{u}$. The resulting image $\tilde{\mathbf{y}}_{u}^{\mathcal{S}}$ serves as an estimate of the original image; hence, $\hat{\mathbf{x}}(\mathbf{y})=\tilde{\mathbf{y}}_{u}^{\mathcal{S}}$. The denoising process is summarized in Figure I

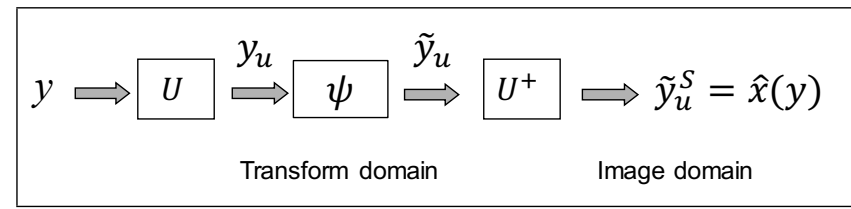

Fig. 1. The shrinkage denoising in the transform domain.

The performance of shrinkage denoising is intimately dependent on two factors. The first factor is related to the choice of the shrinkage functions (SF) $\left\{\psi_{i}\right\}$ applied to the transform coefficients. The justification for applying a marginal (scalar) SF to each coefficient independently emerges from 
the independence assumption of the wavelet coefficients when the transform is unitary. Assuming the statistical distribution of a wavelet band is stationary, and using the independence assumption of the wavelet coefficients, the SFs for all coefficients in a particular wavelet band can be shown to be identical [12]-[14]. Therefore, if the wavelet transform $U$ is composed of $K$ bands, only $K$ SFs need to be estimated, when $\tilde{\mathbf{y}}_{u}[i]=\psi_{\text {band }(i)}\left(\mathbf{y}_{u}[i]\right)$, where $\operatorname{band}(i)$ indicates the band index of pixel $i$. In principle, having a marginal prior distribution for a wavelet band, the associated SF can be derived using Bayesian estimation (e.g., [12], [14]). Alternatively, the SFs can be learnt directly from the noisy input [1], [10], [11] or from a set of example images that are given offline along with their clean counterparts [15]-[17].

The second factor that influences the denoising performance is the transform used during the process. Although the shrinkage approach using unitary wavelet transforms provides good results, significant improvement is achieved when implementing this technique with redundant transforms. Such transforms include preselected bases such as the undecimated wavelets [18], steerable wavelets [19], and other suggested transforms [20]-[25], or generated transforms that are adaptively learnt from the noisy image [26]-[29]. Note that scalar SFs can no longer be justified in redundant bases, as the transform coefficients are mutually dependent due to the transform redundancy. Nevertheless, the superior results of applying scalar SFs in the over-complete case suggest that such a scheme is still very effective in addition to its appealing efficiency.

The above-mentioned two factors influencing the denoising performance, namely, the transform used and the applied SFs, are mutually dependant and cannot be treated independently. The type of transform used directly influences the shape of the optimal SFs. Moreover, optimal SFs for a redundant transform, such as an undecimated wavelet, are shown to differ from the SFs optimally designed for the unitary basis [13], [26], [30]. To clarify, consider finding the optimal SFs for the unitary case with respect to the MMSE criterion. In other words, try finding a $\psi$ that minimizes

$$
\Delta=E\left\{\|\hat{\mathbf{x}}(\mathbf{y})-\mathbf{x}\|^{2}\right\}
$$

when we have determined that $\hat{\mathbf{x}}(\mathbf{y})=U^{T} \psi(U \mathbf{y})$, the norm $\|\cdot\|$ stands for the $\ell_{2}$ norm and $E\{\cdot\}$ indicates the expectation taken over $\mathbf{x}$ and $\mathbf{y}$. Whenever $U$ is unitary, this minimization can be formulated equivalently in the transform domain (since $\left.U U^{T}=U^{T} U=I\right)$ as:

$$
E\left\{\left\|U^{T} \psi(U \mathbf{y})-\mathbf{x}\right\|^{2}\right\}=E\left\{\|\psi(U \mathbf{y})-U \mathbf{x}\|^{2}\right\}
$$

i.e., $\psi$ is optimized so that the noisy transform coefficients $U \mathbf{y}$ should be as close as possible to the transform coefficients of the clean image $U \mathbf{x}$. For an over-complete transform, however, this equality is no longer valid (since $U U^{T} \neq I$ ). This implies that the optimization for $\psi$ should be expressed in the spatial domain, which is the relevant domain in our case. Because the inverse transform couples wavelet coefficients (inside subband and between subbands), spatial domain optimization requires a joint minimization of all SFs simultaneously, and this optimization is far more complicated to apply. In fact, this might be the reason that SFs applyed in redundant bases are commonly borrowed from the unitary case or optimized in the transform domain with no real justification.

In [13], Raphan and Simoncelli showed that as long as the statistics of the image and the noise are stationary, the expected MSE of the denoised image resulting from applying the SFs, $\psi$ in the unitary bases, is always greater or equal to the MSE of the denoised image resulting from applying the same $\psi$ in the redundant basis (by spatially replicating the unitary basis using, e.g., cycle spinning or undecimated subbands [18], [31] ). Note that this property was proven irrespective of the type of applied SFs, $\psi$. They also showed that when working with a redundant basis, there is an advantage in optimizing the SFs (with respect to the expected error) in the spatial domain rather than in the transform domain. This requires, however, optimizing jointly all SFs simultaneously, making the optimization process a demanding task.

In this paper, we extend the results of [13] and establish a complete picture of the interrelations between the transform used, the optimal shrinkage functions, and the domains in which they are optimized. In particular, we show that for subband optimization, where each $\psi_{i}$ is optimized independently, optimizing each SF in the spatial domain is always better than or equal to optimizing the SFs in the transform domain. This option, besides being simple to implement, is proven to outperform the traditional transform domain optimization while avoiding the demanding spatial domain optimization of all SFs simultaneously.

Additionally, for redundant bases, we provide the expected denoising gain we may achieve, relative to the unitary basis, as a function of the basis redundancy. This result allows a user to make a clever decision about the redundancy used by taking into account the expected denoising gain and the computational time allocated for this process.

\section{REDUNDANT VS. UNITARY TRANSFORMS}

A common axiom in image denoising is that denoising applied in redundant bases (cycle-spinning or undecimated wavelets) outperforms the results obtained in unitary transforms [13], [18]. In this section we examine the relationships between the unitary and redundant transforms. In particular, we offer theoretical justification for applying shrinkage denoising in redundant bases. Some of the relationships in this section were already proven in [13] but we repeat them here for clarity and to provide a complete picture of the interrelations between the transformed used and the domain where the MSE is optimized. To be able to compare different transforms on a common basis, we limit our discussion to the unitary basis and their corresponding cycle-spinning transforms. That is to say, the difference between a unitary and a redundant transform is that the former is properly decimated and thus forms a complete basis, while the latter is formed by cycle-spinning the unitary basis.

A common technique for shrinkage denoising in a redundant basis is the cycle-spinning framework [18]. Cycle-spinning is performed by applying a unitary transform on a set of shifted versions of the image, denoising each version independently, then averaging the results after properly shifting them back. 
Since the transform of a spatially shifted image can be applied equivalently by shifting the transform basis (by the same amount but in the opposite direction), the transform can be seen as a redundant transform, composed of a set of shifted versions of the original unitary transform:

$$
\mathbf{y}_{u_{i}}=U S_{i} \mathbf{y}=U_{i} \mathbf{y} \quad i=1 \ldots N
$$

where $S_{i}$ is a (cyclic) shift operator by the $i$-th displacement, and $U_{i}=U S_{i}$ is a unitary transform composed of the wavelet basis after applying the respective shift. The entire transform is constructed by concatenating together all shifted transforms:

$$
\mathbf{y}_{w}=W \mathbf{y}
$$

where the redundant transform is defined as follows:

$$
W=\frac{1}{\sqrt{N}}\left[\begin{array}{c}
U_{1} \\
U_{2} \\
\vdots \\
U_{N}
\end{array}\right]
$$

Note the $W$ is over-complete and tight frame, satisfying $W^{T} W=I$; however, $W W^{T} \neq I$. Since $W W^{T}$ is a projection matrix ${ }^{1}$ it can be shown that this restricts the eigenvalues of $W W^{T}$ to be 1 or 0 . If $W$ is an $m \times n$ matrix $(m>n)$, then there are $n$ eigen-values of 1 , and $m-n$ eigen-values of 0 [32]. Consequently, for any $n \times 1$ vector $\mathbf{x}$ and $m \times 1$ vector $\mathbf{z}$, we have:

$$
\|W \mathbf{x}\|=\|\mathbf{x}\|
$$

and

$$
\left\|W^{T} \mathbf{z}\right\| \leq\|\mathbf{z}\|
$$

Similarly, since $U_{i}$ is unitary, we have $U_{i}^{T} U_{i}=U_{i} U_{i}^{T}=I$ and accordingly:

$$
\left\|U_{i} \mathbf{x}\right\|=\left\|U_{i}^{T} \mathbf{x}\right\|=\|\mathbf{x}\|, \quad \forall i
$$

Denote by $\mathbf{n}=\mathbf{x}-\mathbf{y}$ the contaminated noise in the image domain before denoising. By applying a unitary transform, the error is transformed as well:

$$
U(\mathbf{x}-\mathbf{y})=U \mathbf{n} \doteq \mathbf{n}_{u}
$$

and similarly, by the redundant transform:

$$
W(\mathbf{x}-\mathbf{y})=W \mathbf{n} \doteq \mathbf{n}_{w}
$$

Since the transforms $U$ and $W$ are tight frames, we have (following Equations 9 and 11 ):

$$
\|\mathbf{n}\|=\left\|\mathbf{n}_{u}\right\|=\left\|\mathbf{n}_{w}\right\|
$$

which means that the norm of the error in the transform domain equals its norm in the image domain, and this is true for the unitary as well as the redundant case.

Now, after applying the shrinkage functions $\psi$ to the transform coefficients, the distortion value may change. We define:

$$
\begin{aligned}
& \tilde{\mathbf{n}}_{u}=U \mathbf{x}-\psi(U \mathbf{y}) \text { and similarly } \tilde{\mathbf{n}}_{w}=W \mathbf{x}-\psi(W \mathbf{y}) \\
& { }^{1} \text { A square matrix } A \text { is a projection matrix iff } A A=A .
\end{aligned}
$$

For the unitary case, the distortion is propagated to the image domain via the inverse transform:

$$
U^{T}(U x-\psi(U \mathbf{y}))=U^{T} \tilde{\mathbf{n}}_{u} \doteq \tilde{\mathbf{n}}_{u}^{\mathcal{S}}
$$

and following Equation 11, we have:

$$
\left\|\tilde{\mathbf{n}}_{u}\right\|=\left\|\tilde{\mathbf{n}}_{u}^{\mathcal{S}}\right\|
$$

i.e., after applying the SFs, the MSE distortion in the transform domain is identical to its distortion in the image domain. As we will see next, in redundant transforms this property is not satisfied. In redundant transforms, the error in the image domain is:

$$
W^{T}(W x-\psi(W \mathbf{y}))=W^{T} \tilde{\mathbf{n}}_{w} \doteq \tilde{\mathbf{n}}_{w}^{\mathcal{S}}
$$

Nevertheless, following Equation 10, we have:

$$
\left\|\tilde{\mathbf{n}}_{w}\right\| \geq\left\|\tilde{\mathbf{n}}_{w}^{\mathcal{S}}\right\|
$$

Note that the above relations (Equations 14 and 13 are valid for any shrinkage functions $\psi$ and for any $\mathbf{x}$ and $\mathbf{y}$.

Letting $\mathbf{s}$ be a vector value depending on $\mathbf{x}$ and $\mathbf{y}$, we define the expected RMSE of $\mathbf{s}$ :

$\|\mathbf{s}\|_{E} \doteq \sqrt{E\|\mathbf{s}\|^{2}} \quad$ where $\quad E\|\mathbf{s}\|^{2}=\int\|\mathbf{s}\|^{2} P(\mathbf{x}, \mathbf{y}) d \mathbf{x} d \mathbf{y}$

Since relations 14 and 13 are true for any $\mathbf{x}$ and $\mathbf{y}$, we can rephrase these relations using a statistical point of view:

$$
\left\|\tilde{\mathbf{n}}_{u}\right\|_{E}=\left\|\tilde{\mathbf{n}}_{u}^{\mathcal{S}}\right\|_{E}
$$

and

$$
\left\|\tilde{\mathbf{n}}_{w}\right\|_{E} \geq\left\|\tilde{\mathbf{n}}_{w}^{\mathcal{S}}\right\|_{E}
$$

These relations are illustrated along the two rows of Figure II We now establish the relationships between the unitary and redundant transforms that are indicated in the two columns of Figure III

We first show that in the transform domain, for the two transforms, the expected MSE distortion is equal. This outcome stems from the stationary property of natural images, where it is assumed that the statistical properties of natural images are shift invariant.

Theorem 1. After denoising, the expected MSE distortions in the transform domain are equal for the unitary and for the redundant transforms. In other words, for any given $\psi$ :

$$
\left\|\tilde{\mathbf{n}}_{u}\right\|_{E}=\left\|\tilde{\mathbf{n}}_{w}\right\|_{E}
$$

Proof 1: In Appendix A.

The last theorem leads to a theoretical justification for applying shrinkage denoising in a redundant basis. This is explicitly expressed in the next theorem:

Theorem 2. For any given $\psi$,

$$
\left\|\tilde{\mathbf{n}}_{u}^{\mathcal{S}}\right\|_{E} \geq\left\|\tilde{\mathbf{n}}_{w}^{\mathcal{S}}\right\|_{E}
$$

Proof 2: In Appendix B.

Theorem 2 completes the entire picture of Figure II: In the transform domain, the expected remaining noise, after shrinkage, is identical for the unitary and the redundant (cyclespinning) wavelet transforms for any shrinkage functions. 
When transforming back into the spatial domain, however, the remaining noise is expected to decrease in the redundant transform while staying the same in the unitary transform. This main conclusion suggests that it is preferable to apply shrinkage denoising in a redundant basis rather than in the unitary basis.

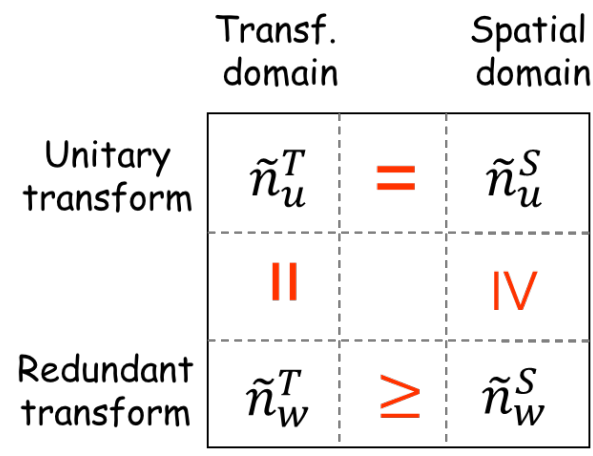

Fig. 2. The expected remaining noise for unitary vs. redundant bases, and spatial vs. transform domains.

\section{OPtIMIZING ThE SHRINKAGE FUnCTIONS}

In this section we deal with the objectives to which the shrinkage functions (SFs) are optimized. As mentioned earlier, SFs play a significant role in the resulting performance, and their optimization is a longstanding topic of study (see, e.g., [26], [33]-[35], just to name a few). In principle, SFs can be derived from the joint statistics of the transform coefficients [13], [33], [34], but, unfortunately, modeling the precise joint statistics is a complicated and still intractable problem. Alternatively, one can optimize the SF of each subband independently using marginal statistics, but as mentioned earlier, this is not optimal in the redundant case. Another option is to learn the optimal SFs from an ensemble of images using a set of noisy and clean examples [13], [36], where the SFs are designed to clean the noisy examples in an optimal manner towards their clean counterparts. As shown next, there are several domains in which the SFs can be optimized in and the resulting quality depends on the selected domain.

In undecimated wavelet transforms, the number of coefficients is $K$ times the size of the image, where $K$ is the number of the wavelet bands. To facilitate the notation for band operations, we reorder the rows of a transform $W$ so that transform rows corresponding to a wavelet band are colocated in a block. Naturally, we extend the same reordering to $\mathbf{y}_{w}$. Assuming we have $K$ different wavelet bands and a corresponding permutation matrix $P$ :

$B=P W=\left[\begin{array}{c}B_{1} \\ B_{2} \\ \vdots \\ B_{K}\end{array}\right]$ and accordingly $\mathbf{y}_{B}=B \mathbf{y}=\left[\begin{array}{c}\mathbf{y}_{1} \\ \mathbf{y}_{2} \\ \vdots \\ \mathbf{y}_{K}\end{array}\right]$

where $\mathbf{y}_{k}=B_{k} \mathbf{y}$ represents the coefficients of the $k^{t h}$ band. The new reordering does not change the tight frame property; thus, if $W^{T} W=I$, we have $B^{T} B=I$ as well. In the new reordering, a vector of SFs, $\psi=\left[\psi_{1}, \psi_{2}, \cdots, \psi_{K}\right]$, can be represented efficiently as follows in Equation 17 Since $\psi_{k}$ is applied similarly to all coefficients in the $k^{t h}$ band, we can rewrite Equation 3 as

$$
\tilde{\mathbf{y}}_{k}=\psi_{k}\left(\mathbf{y}_{k}\right)
$$

which means that the scalar mapping $\psi_{k}: \Re \rightarrow \Re$ is applied individually to each entry in $\mathbf{y}_{k}$. The clean image is then estimated using the adjoint:

$$
\hat{\mathbf{x}}(\mathbf{y})=B^{T} \psi\left(\mathbf{y}_{B}\right)=\sum_{k=1}^{K} B_{k}^{T} \tilde{\mathbf{y}}_{k} \doteq \sum_{k=1}^{K} \tilde{\mathbf{y}}_{k}^{\mathcal{S}}
$$

where we define $\tilde{\mathbf{y}}_{k}^{\mathcal{S}}=B_{k}^{T} \tilde{\mathbf{y}}_{k}$. This process is illustrated in the upper pipeline of Figure III Let the SFs be a set of mapping functions taken from a given function space $\Psi$. The optimal set of SFs with respect to the MSE criterion is then obtained by finding the function set $\psi \subset \Psi$ that minimizes the following objective:

$$
\hat{\psi}=\arg \min _{\psi \in \Psi} \Delta(\psi)
$$

where

$$
\Delta(\psi)=\sqrt{E\left\{\|\mathbf{x}-\hat{\mathbf{x}}(\mathbf{y})\|^{2}\right\}}
$$

where $\hat{\mathbf{x}}(\mathbf{y})$ is as estimate defined in Equation 18 and $E\{\cdot\}$ stands for the expectation taken over $(\mathbf{x}, \mathbf{y})$.

The above minimization is complicated to accomplish as it requires modeling the entire joint statistics of natural images. Below we consider other alternatives for the objective functions. We examine three objectives expressing the optimal set of SFs. We refer to the definitions illustrated in Figure III

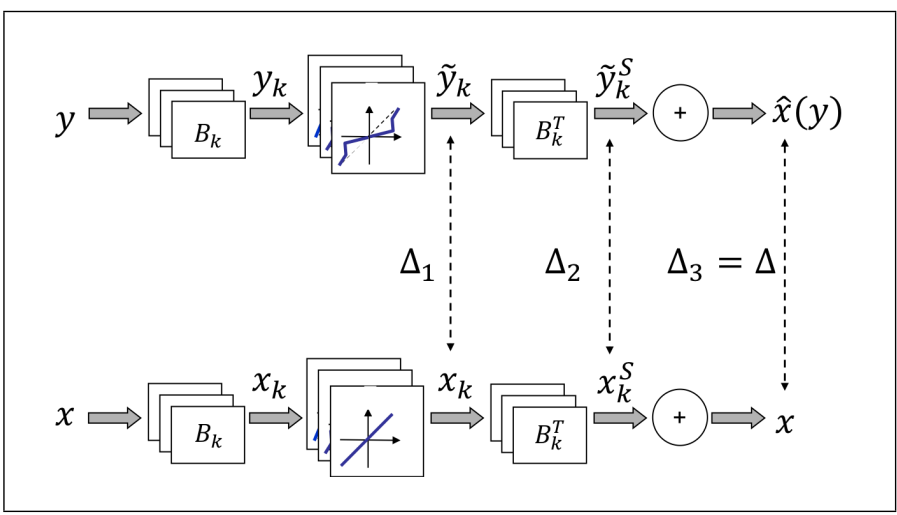

Fig. 3. The denoising process and three optimization schemes for the shrinkage functions.

- Method 1 (transform domain - independent bands): A set of SFs are optimized in the transform domain. The optimization is applied by minimizing the objective function:

$$
\Delta_{1}=\sum_{k} \sqrt{E\left\{\left\|\mathbf{x}_{k}-\tilde{\mathbf{y}}_{k}\right\|^{2}\right\}}
$$

where $\mathbf{x}_{k}=B_{k} \mathbf{x}$ is the clean counterpart of $\tilde{\mathbf{y}}_{k}$. Since this objective is composed of a sum of independent terms, each of which contains a particular band, the minimization of this objective can be applied at each wavelet band 
independently, using only its marginal statistics (since the SFs are scalars); namely:

$$
\hat{\psi}_{k}=\arg \min _{\psi \in \Psi} E\left\{\left\|\mathbf{x}_{k}-\tilde{\mathbf{x}}_{k}\right\|^{2}\right\} \quad \forall k \in\{1,2, . ., K\}
$$

where the expectation is over $\mathbf{y}_{k}, \mathbf{x}_{k}$.

- Method 2 (spatial domain - independent bands): A set of SFs is optimized in the spatial domain. The objective term for this method reads:

$$
\Delta_{2}=\sum_{k} \sqrt{E\left\{\left\|\mathbf{x}_{k}^{\mathcal{S}}-\tilde{\mathbf{y}}_{k}^{\mathcal{S}}\right\|^{2}\right\}}
$$

where $\mathbf{x}_{k}^{\mathcal{S}}=B_{k}^{T} \mathbf{x}_{k}$ and $\tilde{\mathbf{y}}_{k}^{\mathcal{S}}=B_{k}^{T} \tilde{\mathbf{y}}_{k}$. Note that though the objective criterion is expressed in the spatial domain, the SFs can be optimized for each band independently. Thus, although intra-band dependencies are conveyed through the adjoint transform and must be considered, the inter-band dependencies are ignored.

- Method 3 (spatial domain - joint bands): The objective goal is expressed in the spatial domain:

$$
\Delta_{3}=\sqrt{E\left\{\left\|\sum_{k}\left(\mathbf{x}_{k}^{\mathcal{S}}-\tilde{\mathbf{y}}_{k}^{\mathcal{S}}\right)\right\|^{2}\right\}}
$$

It is easy to verify that this objective gives the actual expected error as defined in Equation 19 , thus, $\Delta_{3}=$ $\Delta$. In this scheme, the SFs are evaluated simultaneously while inter-band as well as intra-band dependencies must be taken into account.

Denote the deviation of the approximated coefficients from the clean coefficients by $\mathbf{d}_{k}=\mathbf{x}_{k}-\tilde{\mathbf{y}}_{k}$ and similarly $\mathbf{d}_{k}^{S}=B_{k}^{T}\left(\mathbf{x}_{k}-\tilde{\mathbf{y}}_{k}\right)=B_{k}^{T} \mathbf{d}_{k}$. Using this notation, the above objectives read:

$$
\Delta_{1}=\sum_{k} \sqrt{E\left\{\left\|\mathbf{d}_{k}\right\|^{2}\right\}}, \Delta_{2}=\sum_{k} \sqrt{E\left\{\left\|\mathbf{d}_{k}^{\mathcal{S}}\right\|^{2}\right\}},
$$

and

$$
\Delta_{3}=\sqrt{E\left\{\left\|\sum_{k} \mathbf{d}_{k}^{\mathcal{S}}\right\|^{2}\right\}}=\Delta
$$

For each method defined above, denote an associated optimal SF, $\hat{\psi}^{i}$, as follows:

$$
\hat{\psi}^{i}=\arg \min _{\psi \in \Psi} \Delta_{i}, \quad \text { for } i=1 . .3
$$

Additionally, the objective $\Delta\left(\hat{\psi}^{i}\right)$ denotes the actual expected error as defined in Equation 19 when applying the SF $\hat{\psi}^{i}$. In the following, we show that if the wavelet transform is unitary, then all three methods produce the same result. This is illustrated in the upper line of Figure 5

Theorem 3. For the unitary case we have:

$$
\Delta\left(\hat{\psi}^{1}\right)=\Delta\left(\hat{\psi}^{2}\right)=\Delta\left(\hat{\psi}^{3}\right)
$$

Proof 3: To show the above relations, we prove that actually, for the unitary case, $\hat{\psi}^{1}=\hat{\psi}^{2}=\hat{\psi}^{3}$, which derives the theorem. Recall that $\hat{\psi}=\left[\hat{\psi}_{1} \cdots \hat{\psi}_{K}\right]$ is composed of $K \mathrm{SFs}$, each of which applies to a particular band. Thus, $\mathbf{d}_{k}$ and $\mathbf{d}_{k}^{\mathcal{S}}$ depend only on $\psi_{k}$, and we can apply the optimization to each band independently. For the $k^{t h}$ band, we have:

$$
\hat{\psi}_{k}^{1}=\arg \min _{\psi_{k}} E\left\{\left\|\mathbf{d}_{k}\right\|^{2}\right\}
$$

and

$$
\psi_{k}^{2}=\arg \min _{\psi_{k}} E\left\{\left\|B_{k}^{T} \mathbf{d}_{k}\right\|^{2}\right\}
$$

Since $W$ is unitary, $W W^{T}=I$, and accordingly, $B_{i} B_{j}^{T}=$ $\delta_{i, j} I$. Using this relation we get:

$$
\left\|B_{k}^{T} \mathbf{d}_{k}\right\|^{2}=\left\|\mathbf{d}_{k}\right\|^{2}
$$

which gives

$$
\hat{\psi}_{k}^{1}=\hat{\psi}_{k}^{2}, \quad \text { for } \quad k=1 . . K
$$

and accordingly $\hat{\psi}^{1}=\hat{\psi}^{2}$, which implies the first relation in the theorem.

Similarly,

$$
\hat{\psi}_{k}^{3}=\arg \min _{\psi_{k}} E\left\{\left\|\sum_{j} B_{j}^{T} \mathbf{d}_{j}\right\|^{2}\right\}
$$

However,

$$
\left\|\sum_{j} B_{j}^{T} \mathbf{d}_{j}\right\|^{2}=\left(\sum_{i} \mathbf{d}_{i}^{T} B_{i}\right)\left(\sum_{j} B_{j}^{T} \mathbf{d}_{j}\right)=\sum_{j}\left\|\mathbf{d}_{j}\right\|^{2}
$$

Thus,

$$
\hat{\psi}_{k}^{3}=\arg \min _{\psi_{k}} E\left\{\sum_{j}\left\|\mathbf{d}_{j}\right\|^{2}\right\}=\arg \min _{\psi_{k}} E\left\{\left\|\mathbf{d}_{k}\right\|^{2}\right\}=\hat{\psi}_{k}^{1}
$$

where $k \in\{1 . . K\}$. This yields $\hat{\psi}^{3}=\hat{\psi}^{1}$, which implies the second relation of the theorem. Hence, in the unitary case, optimizing the SFs using any one of the above methods is equivalent

Theorem 3 establishes the justification for optimizing the $\mathrm{SFs}$ in the transform domain, in cases where the transform used is unitary. Using Method 1, each individual SF can be optimized independently, collecting only marginal statistics. This property makes this scheme very appealing and thus very popular (e.g., [10], [18], [19]). Theorem 4 shows that in the over-complete transform, the situation is totally different, and the domain in which we apply the optimization makes a difference (see Figure 4 for an illustration).

Theorem 4. Let the transform $W$ be over-complete and tight frame. In such a case, for each $\psi$,

$$
\Delta_{1}(\psi) \geq \Delta_{2}(\psi) \geq \Delta_{3}(\psi)
$$

Proof 4: Since $W$ is tight frame, it follows that $W^{T} W=I$. It can easily be shown that this restricts the norm of each $B_{k}:\left\|B_{k}\right\|=\lambda_{(k)} \leq 1$, where $\lambda_{(k)}$ denotes the maximal 
eigen-value of $B_{k} B_{k}^{T}$ [32]. This yields that for any vector $\mathbf{z},\left\|B_{k}^{T} \mathbf{z}\right\| \leq\|\mathbf{z}\|$; hence,

$$
\begin{aligned}
\Delta_{1} & =\sum_{j} \sqrt{E\left\{\left\|\mathbf{d}_{j}\right\|^{2}\right\}} \geq \sum_{j} \sqrt{E\left\{\left\|B_{j}^{T} \mathbf{d}_{j}\right\|^{2}\right\}} \\
& =\sum_{j} \sqrt{E\left\{\left\|\mathbf{d}_{j}^{\mathcal{S}}\right\|^{2}\right\}}=\Delta_{2}
\end{aligned}
$$

and this proves the first inequality. Due to the triangular inequality of a norm ${ }^{2}$, we also have:

$$
\Delta_{2}=\sum_{j} \sqrt{E\left\{\left\|\mathbf{d}_{j}^{\mathcal{S}}\right\|^{2}\right\}} \geq \sqrt{E\left\{\left\|\sum_{j} \mathbf{d}_{j}^{\mathcal{S}}\right\|^{2}\right\}}=\Delta_{3}
$$

which gives the second inequality in the theorem

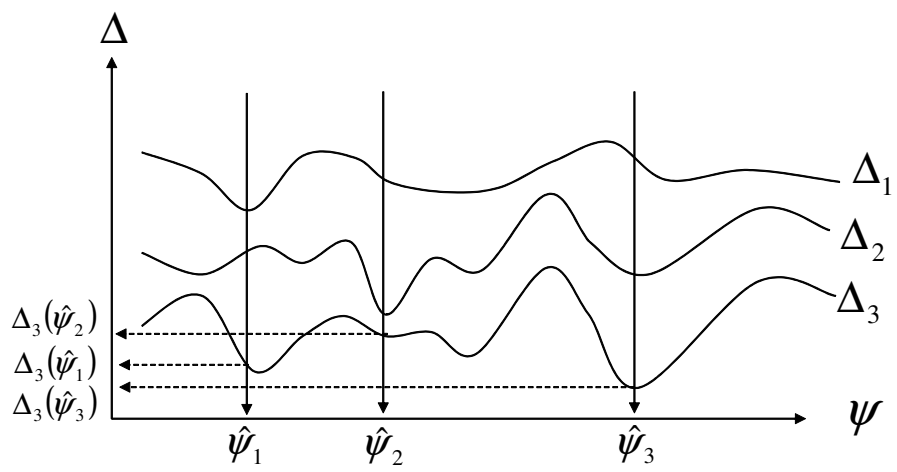

Fig. 4. Illustrated profiles of penalties following Theorem 4 The $\mathrm{x}$-axis indicates various $\psi$ values. Note that in this example, $\Delta\left(\hat{\psi}^{\mathrm{T}}\right)<\Delta\left(\hat{\psi}^{2}\right)$

Theorem 4 reveals that in the over-complete case, optimizing the SFs in the transform domain is not optimal. In the following we provide justification for optimizing the SFs using Methods 1 and 2 as they provide upper bounds for the desired penalty ( $\Delta$ of Method 3), that might be difficult to achieve.

Theorem 5. Let $\hat{\psi}^{i}=\arg \min _{\psi \in \Psi} \Delta_{i}(\psi)$ as defined in Equation 21 In the over-complete case,

$$
\Delta_{1}\left(\hat{\psi}^{1}\right) \geq \Delta_{2}\left(\hat{\psi}^{2}\right) \geq \Delta_{3}\left(\hat{\psi}^{3}\right)
$$

Proof 5: The SF $\hat{\psi}^{3}$ minimizes $\Delta_{3}$; thus, $\Delta_{3}\left(\hat{\psi}^{2}\right) \geq \Delta_{3}\left(\hat{\psi}^{3}\right)$. Following Theorem 4. however, we have that $\Delta_{2}\left(\hat{\psi}^{2}\right) \geq \Delta_{3}\left(\hat{\psi}^{2}\right)$, from which it readily follows that $\Delta_{2}\left(\hat{\psi}^{2}\right) \geq \Delta_{3}\left(\hat{\psi}^{3}\right)$. The second inequality can be shown using a similar argument. Q.E.D.

Note also that according to the proof above, the actual errors (i.e., $\Delta_{3}=\Delta$ ) using $\hat{\psi}^{2}$ and $\hat{\psi}^{1}$ are even tighter, i.e.:

$$
\begin{array}{ll}
\Delta_{2}\left(\hat{\psi}^{2}\right) \geq \Delta\left(\hat{\psi}^{2}\right) \geq \Delta\left(\hat{\psi}^{3}\right) & \text { for Method } 2 \\
\Delta_{1}\left(\hat{\psi}^{1}\right) \geq \Delta\left(\hat{\psi}^{1}\right) \geq \Delta\left(\hat{\psi}^{3}\right) & \text { for Method } 1
\end{array}
$$

and since $\Delta_{1}\left(\hat{\psi}^{1}\right) \geq \Delta_{2}\left(\hat{\psi}^{2}\right)$ (Theorem 5), the SF $\hat{\psi}^{2}$ has a better bound than $\hat{\psi}^{1}$. Thus, it is expected that $\Delta\left(\hat{\psi}^{1}\right) \geq$ $\Delta\left(\hat{\psi}^{2}\right)$. Nevertheless, it cannot be assured that the actual error for $\hat{\psi}_{2}$ outperforms the actual error of $\hat{\psi}_{1}$, i.e., the relation:

$$
\Delta\left(\hat{\psi}^{1}\right) \geq \Delta\left(\hat{\psi}^{2}\right)
$$

${ }^{2}$ Note that the expectation value can be inserted into the norm definition. is not necessarily true. To prove this, see a counter-example in Figure 4.

To conclude, in redundant transforms $\Delta_{3}=\Delta$ determines the actual error and it is the optimal penalty to minimize. Nevertheless, since it requires inter- and intra-bands statistics, it is sometimes complicated to optimize. $\Delta_{1}$ is the easiest term to minimize as it requires collecting only marginal statistics. Indeed, this approach is commonly used in the traditional techniques (hard/soft thresholding originated from this penalty). $\Delta_{2}$ is a better penalty to minimize than $\Delta_{1}$ as its bound is tighter, although it might be harder to optimize as it requires modeling intra-band statistics. Nevertheless, it is not guaranteed that $\Delta\left(\hat{\psi}^{1}\right) \geq \Delta\left(\hat{\psi}^{2}\right)$. Thus, there is an inherent trade-off between the three methods, while spatial domain optimization (Method 3) is preferable with respect to denoising quality, transform domain optimization (Method 1) is the most efficient to apply. Weak spatial domain (Method 2 ) is a good compromise between quality and efficiency.

In unitary transforms, all optimization objectives (Methods 1, 2, and 3) will generate similar results. Using Theorem 2 above, however, it was proven that it is expected that denoising in redundant transforms will generate better results than using unitary transforms. These relations are summarized in Figure 5

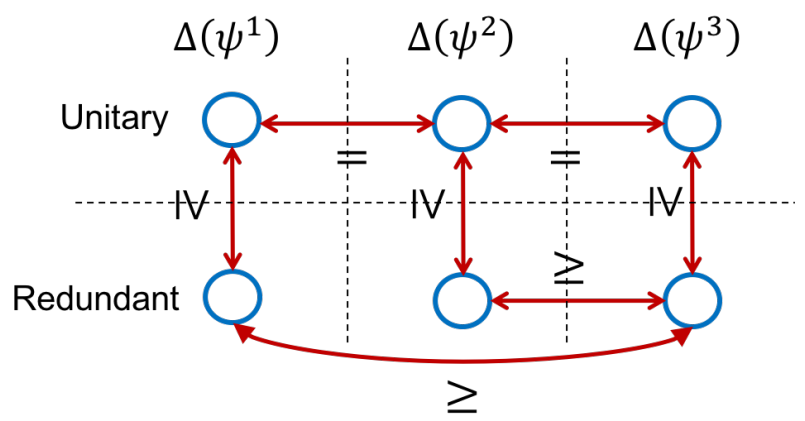

Fig. 5. The relationships of the expected error, $\Delta(\psi)$, when applying shrinkage functions that have been optimized using penalty $1 . .3$ and in unitary vs. redundant transforms.

\section{IMPROVEMENT RATES FOR REDUNDANT TRANSFORMS}

In this section we analyze the expected improvement of the remaining MSE with respect to the redundant rate of the transform used. We assume an over-complete wavelet transform made by cycle spinning as given in Equation 7 .

$$
\mathbf{y}_{w}=W \mathbf{y}
$$

where $W$ is composed of $k$ shifted versions of the unitary transform $U$.

$$
W=\frac{1}{\sqrt{k}}\left[\begin{array}{c}
U_{1} \\
U_{2} \\
\vdots \\
U_{k}
\end{array}\right]
$$

The redundancy rate of this transform is $k$, where $k \in$ $\{1 \cdots n\}$. Namely, each subband has $k$ shifts of the corresponding basis function. Note that if $k=1$, the transform reduces to the unitary transform while the maximal redundancy 
is when $k=n$. The transform is composed of a $k n \times n$ matrix $W$, and is tight frame $\left(W^{T} W=I\right)$. Denote by $\operatorname{Col}(W)$ the column space of $W$ (see Figure 6). The column space forms an $n$-dimensional subspace embedded in $\mathbb{R}^{k n}$. Since $W$ is tight frame, it can easily be verified that the distance between two vectors in the transform domain that are in $\operatorname{Col}(W)$ is identical to their distance in the spatial domain; i.e., let $\mathbf{z}_{1}=W \mathbf{x}_{1}$ and $\mathbf{z}_{2}=W \mathbf{x}_{2}$. The vectors $\mathbf{z}_{1}$ and $\mathbf{z}_{2}$ are in $\operatorname{Col}(W)$; thus,

$$
\left\|\mathbf{x}_{1}-\mathbf{x}_{2}\right\|=\left\|\mathbf{z}_{1}-\mathbf{z}_{2}\right\|
$$

On the other hand, if $\mathbf{z}_{1}$ and $\mathbf{z}_{2}$ are two vectors in the transform domain that are not in $\operatorname{Col}(W)$, their distance in the spatial domain is identical to their distance in the transform domain after projecting onto $\operatorname{Col}(W)$. Namely, if $\mathbf{x}_{1}=W^{T} \mathbf{z}_{1}$ and $\mathbf{x}_{2}=W^{T} \mathbf{z}_{2}$ (i.e., $\mathbf{z}_{1}, \mathbf{z}_{2}$ are two vectors in the transform domain, and $\mathbf{x}_{1}, \mathbf{x}_{2}$ are their counterparts in the spatial domain), then

$$
\left\|\mathbf{x}_{1}-\mathbf{x}_{2}\right\|=\left\|P\left(\mathbf{z}_{1}\right)-P\left(\mathbf{z}_{2}\right)\right\|
$$

where $P(\mathbf{z})=W W^{T} \mathbf{z}$ is the projection of vector $\mathbf{z}$ onto $\operatorname{Col}(W)$.

Denote by $\|\mathbf{n}\|=\|\mathbf{y}-\mathbf{x}\|$ and $\left\|\mathbf{n}_{w}\right\|=\left\|\mathbf{y}_{w}-\mathbf{x}_{w}\right\|$ the RMSE between $\mathbf{x}$ and $\mathbf{y}$ in the spatial domain and between $\mathbf{x}_{w}$ and $\mathbf{y}_{w}$ in the transform domain, respectively, as defined in Section II Since both vectors, $\mathbf{x}_{w}$ and $\mathbf{y}_{w}$, are in $\operatorname{Col}(W)$, the above relations give readily that $\|\mathbf{n}\|=\left\|\mathbf{n}_{w}\right\|$. This was also verified in Equation 12 above. After applying the shrinkage functions $\tilde{\mathbf{y}}_{w}=\psi\left(\mathbf{y}_{w}\right)$, however, the signal $\tilde{\mathbf{y}}_{w}$ is not necessarily in $\operatorname{Col}(W)$. Denote by $\mathbf{y}^{*}$ the optimal possible reconstruction result ${ }^{3}$ and its representation in the transform domain by $\mathbf{y}_{w}^{*}=W \mathbf{y}^{*}$. Clearly $\mathbf{y}_{w}^{*} \in \operatorname{Col}(W)$ and accordingly, the optimal reconstructed RMSE is:

$$
\left\|\mathbf{n}^{*}\right\|=\left\|\mathbf{y}^{*}-\mathbf{x}\right\|=\left\|\mathbf{y}_{w}^{*}-\mathbf{x}_{w}\right\|
$$

The shrinkage functions, however, provide $\tilde{\mathbf{y}}_{w}$, which deviates from $\mathbf{y}_{w}^{*}$ by $\tilde{\mathbf{r}}_{w}$ (see Figure 6):

$$
\tilde{\mathbf{r}}_{w}=\tilde{\mathbf{y}}_{w}-\mathbf{y}_{w}^{*}
$$

Theorem 6. For an over-complete transform with redundancy $k$, the expected RMSE is bounded from above by:

$$
\left\|\tilde{\mathbf{n}}_{w}^{S}\right\|_{E} \leq\left\|\mathbf{n}^{*}\right\|_{E}+\frac{1}{\sqrt{k}}\left\|\tilde{\mathbf{r}}_{w}\right\|_{E}
$$

where $\Delta=\left\|\tilde{\mathbf{n}}_{w}^{S}\right\|_{E}$ is the resulting RMSE in the spatial domain. In other words, the larger the redundancy, the closer the resulting RMSE is to the optimal one and the convergence rate goes like $1 / \sqrt{k}$.

Proof 6: Recall that

$$
\begin{array}{r}
\mid \tilde{\mathbf{n}}_{w}^{\mathcal{S}}\left\|_{E} \doteq\right\| \tilde{\mathbf{y}}_{w}^{S}-\mathbf{x}\left\|_{E}=\right\| P\left(\tilde{\mathbf{y}}_{w}\right)-\mathbf{x}_{w} \|_{E}= \\
=\left\|P\left(\mathbf{y}_{w}^{*}+\tilde{\mathbf{r}}_{w}\right)-\mathbf{x}_{w}\right\|_{E}=\left\|\mathbf{y}_{w}^{*}+P\left(\tilde{\mathbf{r}}_{w}\right)-\mathbf{x}_{w}\right\|_{E}
\end{array}
$$

\footnotetext{
${ }^{3}$ The solution is biased as we apply scalar mapping functions whereas the optimal mapping function should be a scalar field [13].
}

where the second equality is due to Equation 29 and the fourth equality is due to the fact that $\mathbf{y}_{w}^{*} \in \operatorname{Col}(W)$. Using the triangular inequality, we get:

$$
\left\|\mathbf{y}_{w}^{*}+P\left(\tilde{\mathbf{r}}_{w}\right)-\mathbf{x}_{w}\right\| \leq\left\|\mathbf{y}_{w}^{*}-\mathbf{x}_{w}\right\|+\left\|P\left(\tilde{\mathbf{r}}_{w}\right)\right\|=\left\|\mathbf{n}^{*}\right\|+\left\|P\left(\tilde{\mathbf{r}}_{w}\right)\right\|
$$

Thus,

$$
\left\|\tilde{\mathbf{n}}_{w}^{\mathcal{S}}\right\| \leq\left\|\mathbf{n}^{*}\right\|+\left\|P\left(\tilde{\mathbf{r}}_{w}\right)\right\|
$$

We follow the same argument that was used in Appendix A where we showed that due to the stationarity of natural images, for any $W$ (any redundancy rate), we have: $\left\|\tilde{\mathbf{n}}_{w}\right\|_{E}=\left\|\tilde{\mathbf{n}}_{u}\right\|_{E}$ where $\tilde{\mathbf{n}}_{w}=\tilde{\mathbf{y}}_{w}-\mathbf{x}_{w}$ and $\tilde{\mathbf{n}}_{u}=\tilde{\mathbf{y}}_{u}-\mathbf{x}_{u}$. This argument also holds if we switch $\mathbf{x}$ with $\mathbf{y}^{*}$ providing:

$$
\left\|\tilde{\mathbf{r}}_{w}\right\|_{E}=\left\|\tilde{\mathbf{r}}_{u}\right\|_{E}
$$

where $\tilde{\mathbf{r}}_{w}=\tilde{\mathbf{y}}_{w}-\mathbf{y}_{w}^{*}$ and $\tilde{\mathbf{r}}_{u}=\tilde{\mathbf{y}}_{u}-\mathbf{y}_{u}^{*}$. This gives that the expected value of $\left\|\tilde{\mathbf{r}}_{w}\right\|$ is the same for any $W$ of any redundancy rate and it equals $\left\|\tilde{\mathbf{r}}_{u}\right\|$. Thus, the vector $\tilde{\mathbf{r}}_{w} \in \mathbb{R}^{k n}$ can be seen as a random vector in $\mathbb{R}^{k n}$ whose expected length is constant for any $W$. Since $P\left(\mathbf{r}_{w}\right)$ is an orthogonal projection of a random vector from $k n$-dimensional space onto an $n$ dimensional space, the expected length of $P\left(\mathbf{r}_{w}\right)$ is:

$$
\left\|P\left(\tilde{\mathbf{r}}_{w}\right)\right\|_{E}=\frac{1}{\sqrt{k}}\left\|\tilde{\mathbf{r}}_{w}\right\|_{E}
$$

Moreover, the Johnson-Lindenstrauss Lemma shows that $\left\|P\left(\tilde{\mathbf{r}}_{w}\right)\right\|$ is fairly tight concentrated around $\left\|P\left(\tilde{\mathbf{r}}_{w}\right)\right\|_{E}$ (see [37). Combining Equations 33 and 32, we obtain the relation given in Theorem 6. Q.E.D.

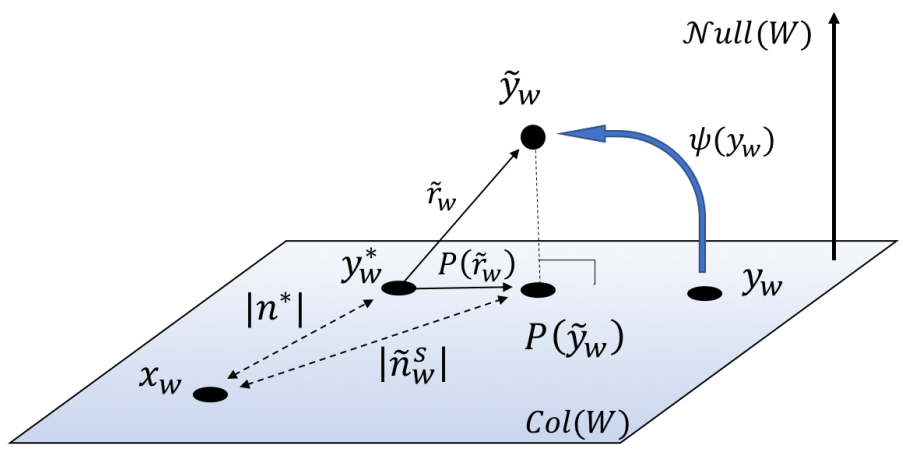

Fig. 6. Due to the triangular inequality, $\left\|\tilde{\mathbf{n}}_{w}^{S}\right\| \leq\left\|\mathbf{n}^{*}\right\|+\left\|P\left(\tilde{\mathbf{r}}_{w}\right)\right\|$. Since $\left\|P\left(\tilde{\mathbf{r}}_{w}\right)\right\|_{E}=\frac{1}{\sqrt{k}}\left\|\tilde{\mathbf{r}}_{w}\right\|_{E}$, Theorem 6 follows.

\section{RESUlts}

In the previous sections we presented three different optimization schemes and their relations. In this section we test the empirical behavior of these methods on real data. The images we used for our experiments can be seen in Fig. 7 .

To test the three methods, we used the optimization scheme suggested in [15]. There the authors suggest modeling the shrinkage functions using piecewise linear mappings:

$$
\psi_{k}\left(\mathbf{y}_{k}\right)=\mathcal{M}_{k}\left(\mathbf{y}_{k} ; \mathbf{p}_{k}\right)
$$

where $\mathbf{p}_{k}$ is a parameter vector controlling the piecewise function. Since the shrinkage function $\mathcal{M}_{k}$ is linear with 

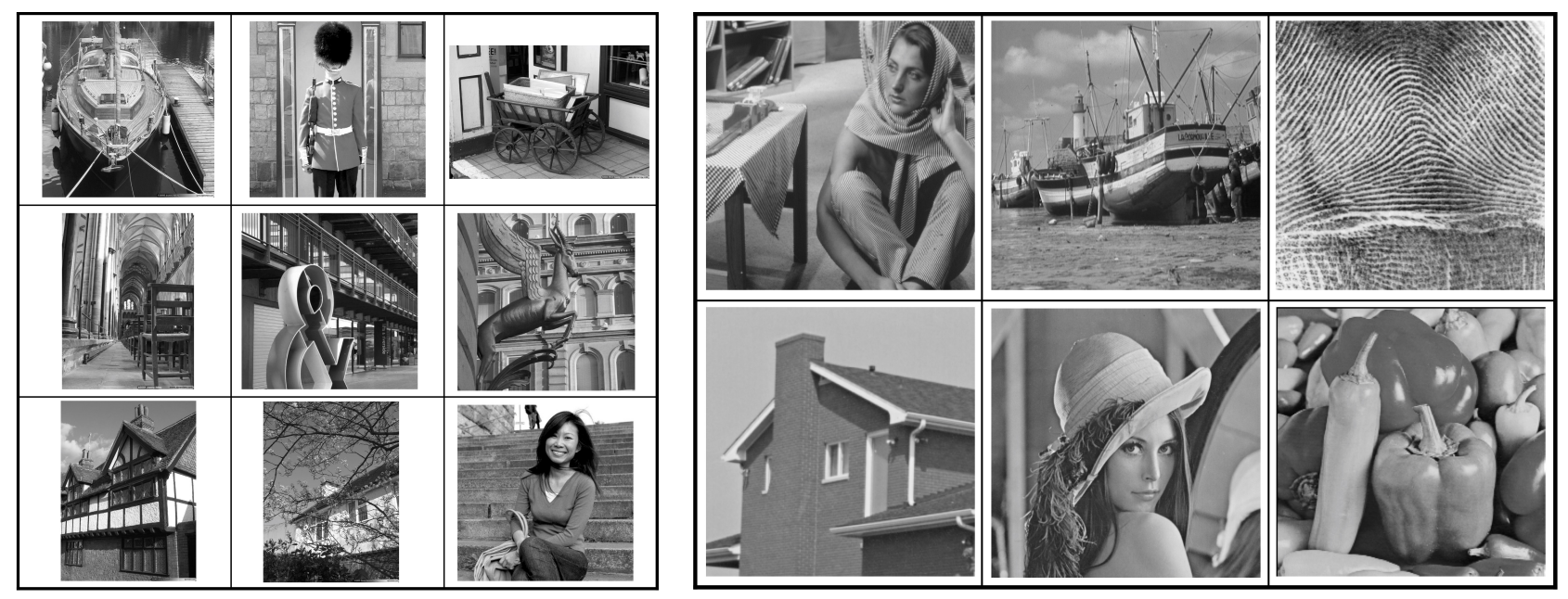

Fig. 7. Left: The images on which the SFs were trained. Right: The images on which the denoising schemes were applied.

respect to the parameter vector $\mathbf{p}_{k}$, optimizing for $\mathbf{p}_{k}$ can be solved in a closed form solution using a set of noisy images along with their clean counterparts. In contrast to the statistical approaches, this technique does not require any estimation of the prior model or of the noise characteristics. The SFs are designed to perform "optimally" with respect to the given examples, under the assumption that they will perform equally well with similar new examples. Using Methods 1 and 2, the optimization is performed on each $\mathbf{p}_{k}$ independently; however, for Method 3, all $\mathbf{p}_{k}$ are optimized simultaneously. For more information about the optimization and the implementation, the reader is referred to [15].

In all the experiments described below, we used the undecimated windowed Discrete Cosine Transform (DCT) as the image transform. Since the DCT transform is unitary, the undecimated DCT is a tight frame. Because of the undecimated form, each wavelet band can be calculated using a single 2D convolution (with the corresponding DCT basis as the convolution kernel). Additionally, the inverse transform can be applied by convolving the rectified coefficients with the kernels forming $B_{k}^{T}$, which are the reflected (180 degree rotation) DCT kernels. More details are given in [15].

\section{A. Comparing all methods for a single noise level}

In the following experiments, unless mentioned otherwise, the setting parameters were defined as follows: (1) Training images were grayscale natural images; a few of them are presented in Figure 7 7 left. (2) Test images were taken from Figure 7-right. (3) Transform basis was the undecimated $8 \times 8$ DCT. (4) The noise consists of additive Gaussian noise with various STD values.

Figure 8 displays some of the SFs obtained for an $8 \times 8$ DCT basis, using the three methods described above for a noise level with a STD $\sigma=20$. SFs on each row correspond to band indices $(i, i)$ of the $8 \times 8$ DCT basis, where $i=2 . .6$ (left to right). Note that a DCT band with an index $(i, j)$ is the result of convolving the image with a DCT basis whose frequency is $i$ along the $x$-axis and $j$ along the $y$-axis. The top, middle and bottom rows show the SFs resulting from the first, second and the third methods, respectively. It can be seen that the SFs of the three methods are different from each other because each case takes into consideration different statistical correlations as explained above.

The obtained SFs were applied to several images 4$]$ shown in Figure 7-right. Figure 9 compares the resulting MSE for each described method. It is composed of six clusters of bars, each of which compares the denoising results of a particular image. Each bar presents the resulting MSE averaged over 10 realizations of noise with a STD $\sigma=20$. The results demonstrate the improvement of the second method over the first method, and the superiority of the third method over the other two. It can be seen that most of the improvement is achieved when applying the objective in the spatial domain (Method 2). Further improvement, although less significant, is achieved when incorporating the band dependencies (Method 3). Note, however, that the resulting MSE of the FINGERPRINT image is better for Method 2 than for Method 3, and this result is incompatible with Equation 23 The reason for this outcome is that the training set for this experiment does not seem to be a good representative of the statistics of the textured FINGERPRINT image. This means that $\hat{\psi}_{2}, \hat{\psi}_{3}$ are not necessarily the right SFs that minimize $\Delta_{2}, \Delta_{3}$, respectively. And, indeed, training the SFs from statisticaly similar images and applying them to the same noisy FINGERPRINT image, provides an MSE value of 89.55 for Method 3, compared to 97.07 in the current plot.

\section{B. Dependencies on the training statistics}

In the next experiments, we tested the relations between the different schemes for various noise levels and for two cases: (a) The training and test sets share the same statistics. (b) The training and test sets have different statistics. Performance was tested for eight different equally spaced noise levels, from STD $\sigma=5$ up to $\sigma=40$. For the first case, we used the same image for training and test sets, where we applied five different noise realizations of the same noise level for each test. This

\footnotetext{
${ }^{4}$ Taken from http://decsai.ugr.es/javier/denoise/test_images/index.htm
} 

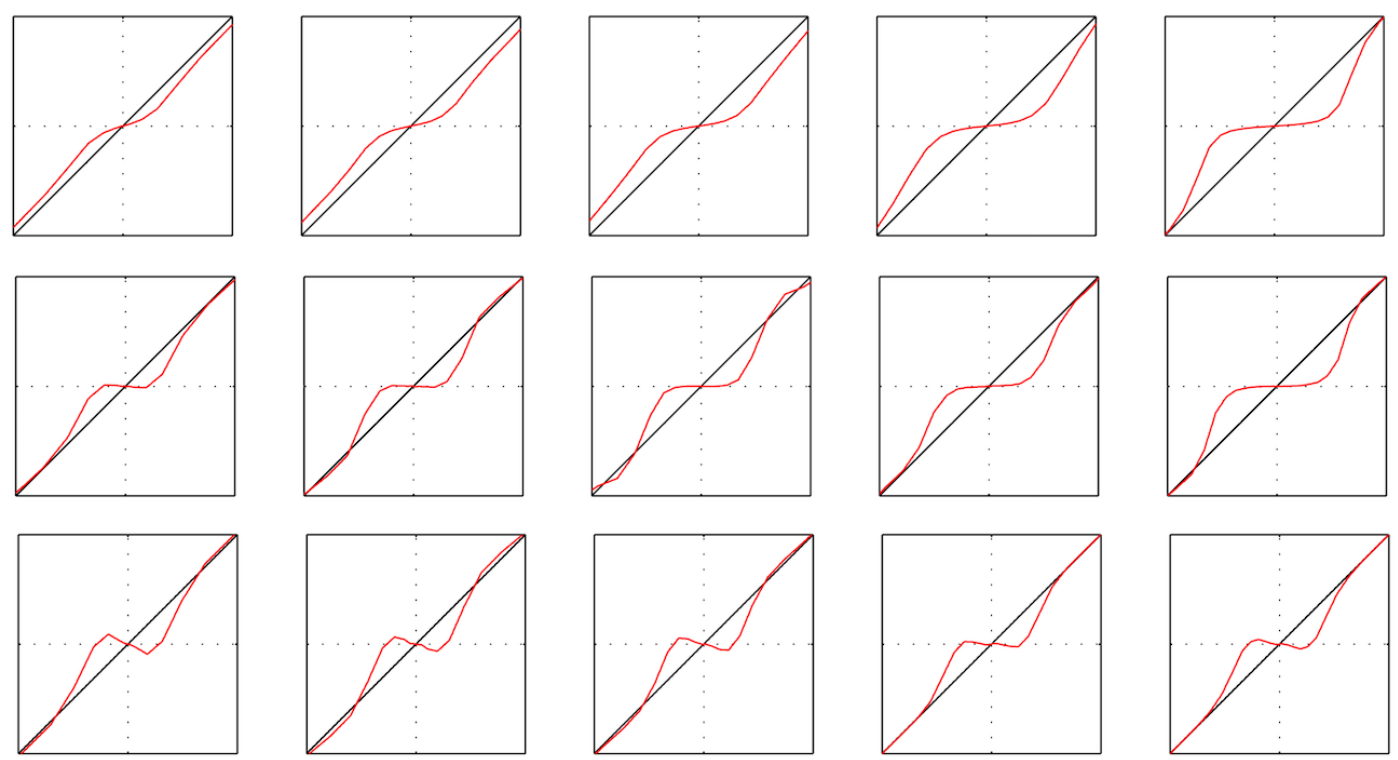

Fig. 8. Comparison of the produced SFs using Method 1 (top row), Method 2 (middle row), and Method 3 (bottom row). SFs on each row correspond to band $(i, i)$ of the $8 \times 8$ DCT basis, where $i=2 . .6$ (left to right). Graph axes are shown in the range [-120,120].

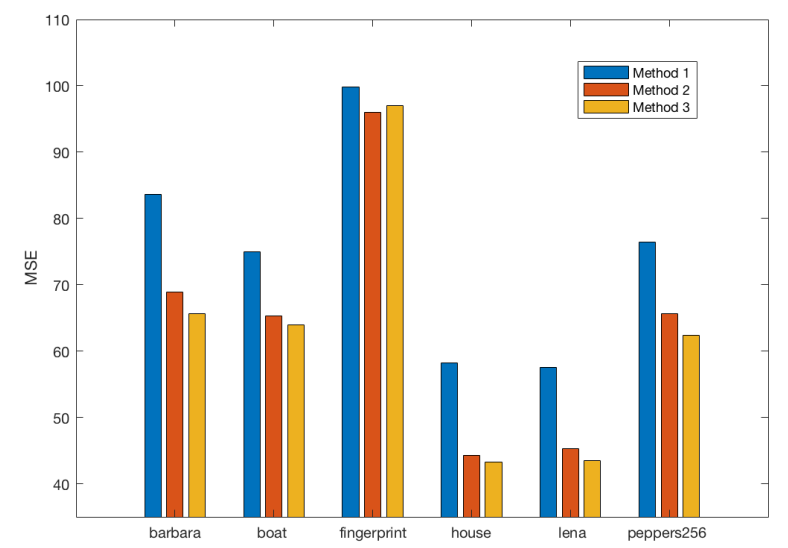

Fig. 9. MSE after applying the SFs produced by Methods 1-3. Each bar is an average over 10 different noise realizations.

was carried out for each image in the set. For the second case, we used a single image (in its turn) as the training set and the remaining images as the test set. The resulting MSEs for eight different noise levels are presented in Fig. 10. The $\mathrm{x}$-axis shows the input MSE and the y-axis presents the output MSE. It can be seen that for both cases, $\Delta\left(\hat{\psi}_{1}\right) \geq \Delta\left(\hat{\psi}_{2}\right) \geq \Delta\left(\hat{\psi}_{3}\right)$. Thus, optimizing the SFs using Method 3 gives the optimal result while optimizing using Method 2 gives better bounds than the traditional optimization using Method 1.

\section{Cost-effective analysis}

Optimizing SFs according to Method 2 offers both tight bounds on the mean MSE distortion and fast implementation. Figures 11-left shows the fraction of deviation of Methods 1 and 2 from the optimal scheme (Method 3). It can be seen that Method 2 deviates on average 4\% relative to Method 3, while Method 1 deviates about $16 \%$.

Figures 11-right shows the computation time taken to train the SFs for each method. It can be seen that training using the optimization scheme of Method 2 takes one quarter of the time needed by Method 3. Thus, Method 2 introduces a costefficient advantage; By allowing a deviation of $4 \%$ from the optimal optimization scheme, we can get a gain, on average, an increase of $70 \%$ in the speed.

\section{Denoising improvements vs. redundancy rate}

To validate the observation about the denoising improvement with respect to the redundancy rate, we measured the resulting MSEs for various redundancy rates. Figure 12 . left shows the MSE for denoising applied to a $13 \times 13$ windowed DCT transform, where the redundancy rates were implemented by shifting the basis functions by $(i, j)$ along the $\mathrm{x}$-axis and the $\mathrm{y}$-axis, where $(i, j) \in\{0,12\} \times\{0,12\}$. Thus, the redundancy rate can range between $k=1$ and $k=169=13 \times 13$. We assume the optimal MSE is given for $k=169$ (maximum redundancy) where in this case $\hat{\mathbf{y}}=\mathbf{y}^{*}$ and $\left\|\mathbf{n}^{*}\right\|=\left\|\mathbf{x}-\mathbf{y}^{*}\right\|=\mathcal{E}_{\text {opt }}$. On the other hand, when $k=1$, we have that $\tilde{\mathbf{y}}_{w} \in \operatorname{Col}(W)$ (in this case $W=U$ ) and thus $\left\|P\left(\tilde{\mathbf{r}}_{w}\right)\right\|=\left\|\tilde{\mathbf{r}}_{u}\right\|=\left\|\tilde{\mathbf{y}}_{u}^{S}-\mathbf{y}^{*}\right\|=\Delta^{*}$. Accordingly, following Theorem 6, the RMSE should be:

$$
R M S E(k) \leq \mathcal{E}_{\text {opt }}+\frac{1}{\sqrt{k}} \Delta^{*}
$$

Figure 12 -left shows the decrease in the measured MSE as a function of the redundancy rate. The solid red curve shows the actual measures while the dashed blue curve shows the expected RMSE following Equation 34 It can be seen that the two plots basically overlap. The measures were taken for the BARBARA image where the noise level was $\sigma=50$. Each MSE measure in this plot is an average of five noise realizations. 


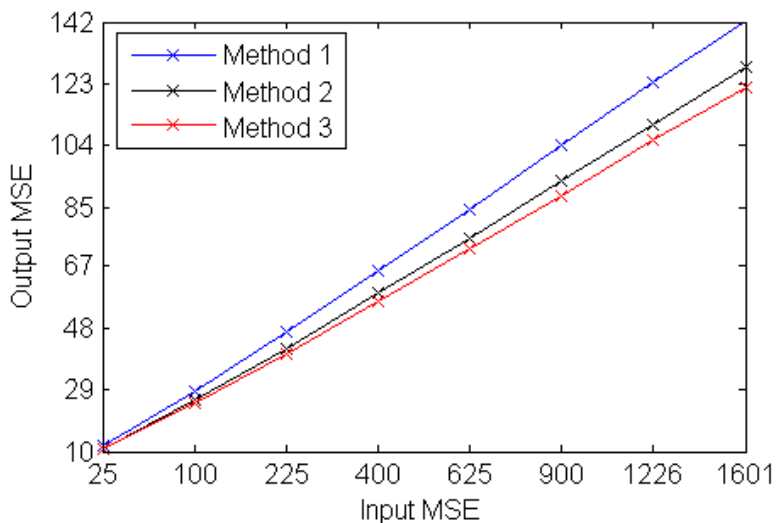

(a)

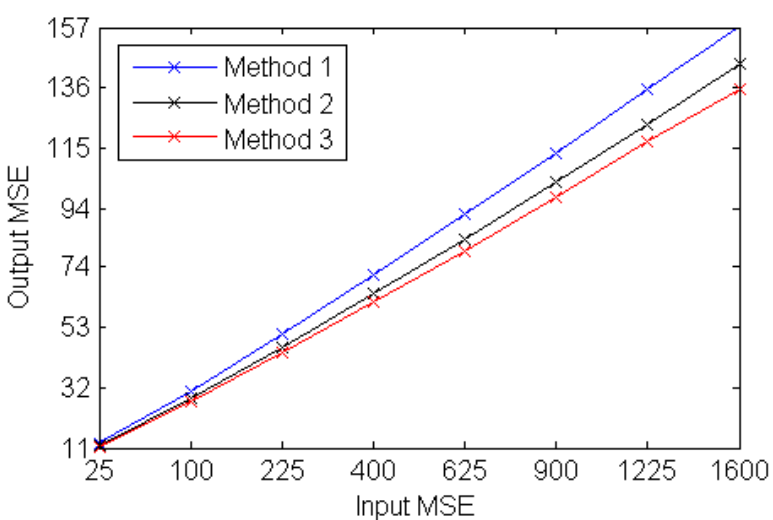

(b)

Fig. 10. Comparison of the different optimization schemes for the case where the training and test images (a) share the same statistics and (b) share different statistics. As expected, for both cases, the optimal optimization scheme is Method 3. The x-axis shows the input MSE and the y-axis presents the output MSE. For the first case, the input and output MSEs were computed from 30 instances of test images, and for the second case, from 180 instances.
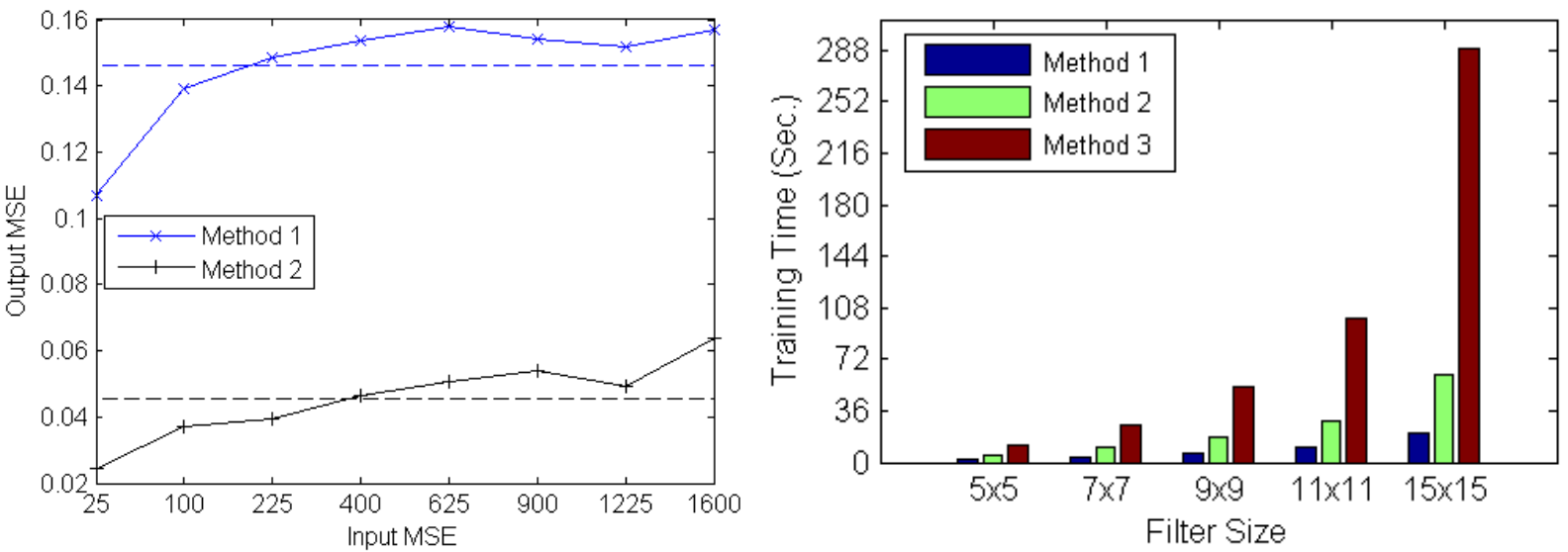

Fig. 11. Left: The plot shows the relative deviation of the output MSE in Methods 1 and 2 relative to Method 3. The x-axis shows the input MSE and the y-axis presents the relative MSE with respect to Method 3. The deviation is approx. 4\% for Method 2 and approx. 16\% for Method 1. Right: This plot shows the training time required for each method.

Figure 12-right shows the optimal achieved MSE for each DCT transform, which were 5, 7,9 and 11 pixels wide. It can be shown that, in most cases, the wider the filter, the more redundancy can achieved and the better the denoising results.

\section{CONCLusions}

Transform denoising using shrinkage functions is a classical framework that is widely used in numerous applications. In this paper we provide a complete picture of the interrelations between the transform used, the optimal shrinkage functions, and the domains in which they are optimized. In particular, we provide a theoretical justification for applying the shrinkage functions in the transform domain and the benefit of applying them in redundant bases.

Additionally, we provided theoretical bounds for the three possible optimization schemes of the shrinkage functions. We showed that for subband optimization, where each $\psi_{i}$ is optimized independently, optimizing the shrinkage function in the spatial domain is always better than or equal to optimizing them in the transform domain. This option, besides being simple to implement, is proven to outperform the traditional transform domain optimization while avoiding the demanding spatial domain optimization of all the shrinkage functions, simultaneously.

For redundant bases, we provided the expected denoising gain we may achieve, relative to the unitary basis, as a function of the basis redundancy. This result allows a user to make a clever decision about the redundancy used by taking into account the expected denoising gain and the computational time allocated for this process.

\section{APPENDIX A}

Theorem 1: After denoising, the expected MSE distortions in the transform domain are equal for the unitary and for the redundant transforms, i.e.:

$$
\left\|\tilde{\mathbf{n}}_{u}\right\|_{E}=\left\|\tilde{\mathbf{n}}_{w}\right\|_{E}
$$

Proof 1: The MSE value $\left\|\tilde{\mathbf{n}}_{u}\right\|_{E}^{2}$ is defined as:

$$
\left\|\tilde{\mathbf{n}}_{u}\right\|_{E}^{2}=E\left\{\left\|\tilde{\mathbf{n}}_{u}\right\|^{2}\right\}=\int\|U \mathbf{x}-\psi\{U \mathbf{y}\}\|^{2} P(\mathbf{x}, \mathbf{y}) d \mathbf{x} d \mathbf{y}
$$



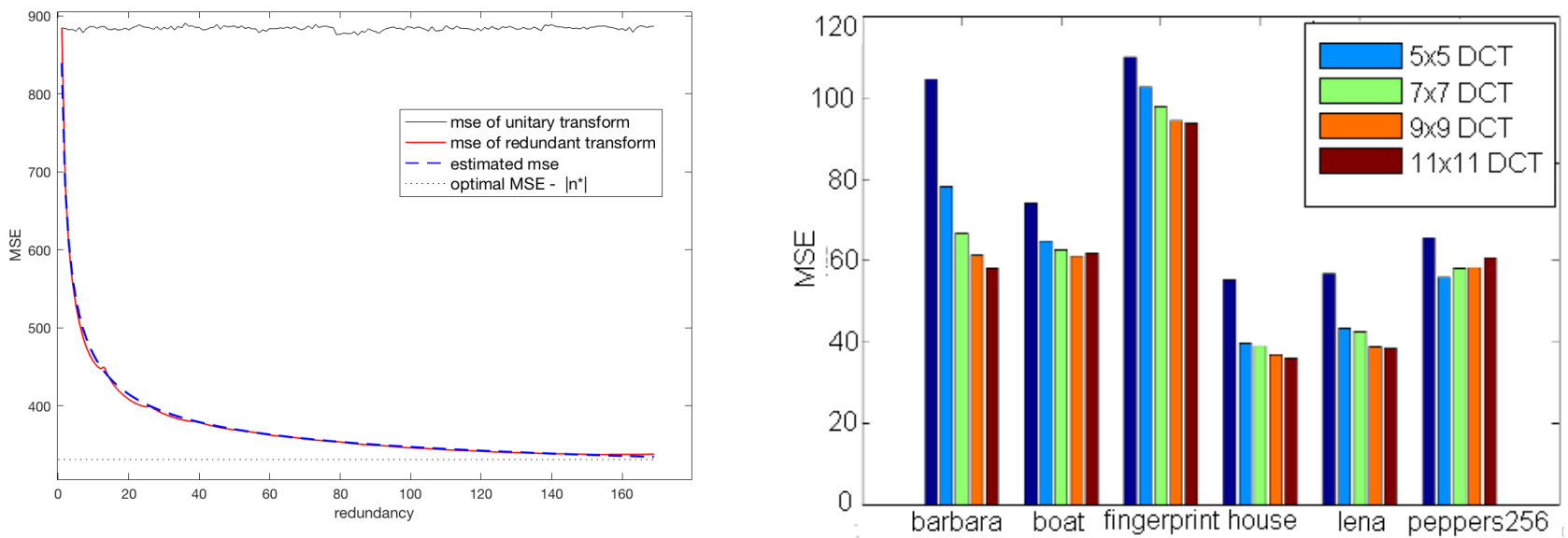

Fig. 12. The performance is improved with the redundancy; The output MSE as a function of the redundancy rate. Left: The filter size was $13 \times 13$ and the noise STD was $\sigma=50$. The plot shows the RMSE as a function of redundancy rate. Right: The wider the filter, the more redundancy achieved and the better the denoising results.

where $P(\mathbf{x}, \mathbf{y})$ denotes the probability distribution function of $(\mathbf{x}, \mathbf{y})$. By changing variables, the above expression can be rewritten as:

$\left\|\tilde{\mathbf{n}}_{u}\right\|_{E}^{2}=\int\left\|U S_{i} \mathbf{x}-\psi\left\{U S_{i} \mathbf{y}\right\}\right\|^{2} P\left(S_{i} \mathbf{x}, S_{i} \mathbf{y}\right)\left|\operatorname{det}\left(S_{i}^{T}\right)\right| d \mathbf{x} d \mathbf{y}$ manuscript.

where $S_{i}$ is a shift operator by the $i^{\text {th }}$ displacement. Now, we exploit the stationary property of natural images. This property gives that for each $S_{i}$ :

$$
P\left(S_{i} \mathbf{x}, S_{i} \mathbf{y}\right)=P(\mathbf{x}, \mathbf{y})
$$

Additionally, we can apply the (adjoint) shift operator to the transform basis, rather than to the images. Using the notation $U S_{i}=U_{i}$ (Equation 66 and having $\left|\operatorname{det}\left(S_{i}^{T}\right)\right|=1$, we get:

$\left\|\tilde{\mathbf{n}}_{u}\right\|_{E}^{2}=\int\left\|U_{i} \mathbf{x}-\psi\left\{U_{i} \mathbf{y}\right\}\right\|^{2} P(\mathbf{x}, \mathbf{y}) d \mathbf{x} d \mathbf{y}=\left\|\tilde{\mathbf{n}}_{u_{i}}\right\|_{E}^{2}$

Now, since $\left\|\tilde{\mathbf{n}}_{w}\right\|_{E}^{2}=\frac{1}{n} \sum_{i=1}^{n}\left\|\tilde{\mathbf{n}}_{u_{i}}\right\|_{E}^{2}$, we conclude:

$\left\|\tilde{\mathbf{n}}_{w}\right\|_{E}^{2}=\frac{1}{n} \sum_{i=1}^{n}\left\|\tilde{\mathbf{n}}_{u_{i}}\right\|_{E}^{2}=\frac{1}{n} \sum_{i=1}^{n}\left\|\tilde{\mathbf{n}}_{u}\right\|_{E}^{2}=\left\|\tilde{\mathbf{n}}_{u}\right\|_{E}^{2} \quad$ Q.E.D.

\section{APPENDIX B}

Theorem 2: For any given $\psi$,

$$
\left\|\tilde{\mathbf{n}}_{u}^{\mathcal{S}}\right\|_{E} \geq\left\|\tilde{\mathbf{n}}_{w}^{\mathcal{S}}\right\|_{E}
$$

Proof 2: After Equation 13 and Theorem 1, we have:

$$
\left\|\tilde{\mathbf{n}}_{u}^{\mathcal{S}}\right\|_{E}=\left\|\tilde{\mathbf{n}}_{u}\right\|_{E}=\left\|\tilde{\mathbf{n}}_{w}\right\|_{E}
$$

However, from Equation 14 it follows that

$$
\left\|\tilde{\mathbf{n}}_{w}\right\|_{E} \geq\left\|\tilde{\mathbf{n}}_{w}^{\mathcal{S}}\right\|_{E}
$$

and therefore:

$$
\left\|\tilde{\mathbf{n}}_{u}^{\mathcal{S}}\right\|_{E} \geq\left\|\tilde{\mathbf{n}}_{w}^{\mathcal{S}}\right\|_{E} \quad \text { Q.E.D. }
$$

\section{ACKNOWLEDGMENT}

The authors would like to thank Prof. Michael Elad for helpful discussions and useful suggestions while writing this

\section{REFERENCES}

[1] D. L. Donoho and I. M. Johnston, "Ideal denoising in an orthonormal basis chosen from a library of bases," C.R. Acad. Sci., vol. 319, pp. $1317-1322,1994$.

[2] H. R. Shahdoosti and Z. Rahemi, "Edge-preserving image denoising using a deep convolutional neural network," Signal Processing, vol. 159, pp. 20-32, 2019.

[3] C. Tian, L. Fei, W. Zheng, Y. Xu, W. Zuo, and C.-W. Lin, "Deep learning on image denoising: An overview," arXiv preprint arXiv:1912.13171, 2019.

[4] H. C. Burger, C. J. Schuler, and S. Harmeling, "Image denoising: Can plain neural networks compete with bm3d?" in 2012 IEEE Conference on Computer Vision and Pattern Recognition. IEEE, 2012, pp. 23922399.

[5] K. Isogawa, T. Ida, T. Shiodera, and T. Takeguchi, "Deep shrinkage convolutional neural network for adaptive noise reduction," IEEE Signal Processing Letters, vol. 25, no. 2, pp. 224-228, 2017.

[6] B. A. Olshausen and D. J. Field, "Emergence of simple-cell receptive field properties by learning a sparse code for natural images," Nature, vol. 381, pp. 607-609, 1996.

[7] —_ "Natural image statistics and efficient coding," Network: Computation in Neural Systems, vol. 7, pp. 333-339, 1996.

[8] J. Hurri, A. Hyv, R. Karhunen, and E. Oja, "Wavelets and natural image statistics," in Proc. Scandinavian Conf. on Image Analysis '97, Lappenranta, Finland, 1998.

[9] S. Mallat, "A theory for multiresolution signal decomposition: The wavelet representation," IEEE Trans. on Pattern Analysis and Machine Intelligence, vol. 11, no. 7, pp. 674-693, July 1989.

[10] D. L. Donoho, "De-noising by soft-thresholding," IEEE Trans. on Information Theory, vol. 41, no. 3, pp. 613-627, 1995.

[11] D. L. Donoho and I. M. Johnston, "Ideal spatial adaptation via wavelet shrinkage," Biometrika, vol. 81, no. 3, pp. 425-455, 1994.

[12] E. P. Simoncelli and E. H. Adelson, "Noise removal via bayesian wavelet coring," in Proceedings of 3rd IEEE International Conference on Image Processing, vol. 1. IEEE, 1996, pp. 379-382.

[13] M. Raphan and E. P. Simoncelli, "Optimal denoising in redundant representations," IEEE Trans. on Image Processing, vol. 17, no. 8, pp. 1342-1352, 2008.

[14] E. P. Simoncelli, "Bayesian denoising of visual images in the wavelet domain," in Bayesian Inference in Wavelet Based Models. Springer, 1999, pp. 291-308. 
[15] Y. Hel-Or and D. Shaked, "A discriminative approach for wavelet denoising," IEEE Trans. on Image Processing, vol. 17, no. 4, pp. 443457, 2008.

[16] A. Adler, Y. Hel-Or, and M. Elad, "A weighted discriminative approach for image denoising with overcomplete representations," in 2010 IEEE International Conference on Acoustics, Speech and Signal Processing. IEEE, 2010, pp. 782-785.

[17] J. Sun and Z. Xu, "Color image denoising via discriminatively learned iterative shrinkage," IEEE Trans. on Image Processing, vol. 24, no. 11 , pp. 4148-4159, 2015.

[18] R. R. Coifman and D. L. Donoho, "Translation invariant de-noising," in Wavelets and Statistics, A. Antoniadis and G. Oppenheim, Eds. Springer-Verlag, 1995, pp. 125-150.

[19] E. P. Simoncelli and E. H. Adelson, "Noise removal via Bayesian wavelet coring," in Third Int'l Conf on Image Proc, vol. I. Lausanne: IEEE Sig Proc Society, 1996, pp. 379-382.

[20] E. J. Candes, "Harmonic analysis of neural networks," Applied and Computational Harmonic Analysis, vol. 6, pp. 197-218, 1999.

[21] P. Carré and D. Helbert, "Ridgelet decomposition: Discrete implementation and color denoising." in Wavelet Applications in Industrial Processing III. Boston, Massachusetts, USA: SPIE, October 2005.

[22] N. Nezamoddini-Kachouie, P. Fieguth, and E. Jernigan, "Bayesshrink ridgelets for image denoising," in Proc. ICIAR 2004, Porto, Portugal, September 2004.

[23] M. N. Do and M. Vetterli, "The contourlet transform: an efficient directional multiresolution image representation," IEEE Trans. Image Processing, to appear

[24] B. Matalon, M. Elad, and M. Zibulevsky, "Image denoising with the contourlet transform," in Proceedings of SPARSE'05, Rennes, France, November 2005.

[25] J. Starck, E. Candes, and D. Donoho, "The curvelet transform for image denoising," IEEE Trans. Image Processing, vol. 11, no. 6, pp. 670-684, June 2002.

[26] M. Elad and M. Aharon, "Image denoising via sparse and redundant representations over learned dictionaries," IEEE Trans. on Image processing, vol. 15, no. 12, pp. 3736-3745, 2006.

[27] W. Dong, X. Li, L. Zhang, and G. Shi, "Sparsity-based image denoising via dictionary learning and structural clustering," in CVPR 2011. IEEE, 2011, pp. 457-464.

[28] H. Krim, D. Tucker, S. Mallat, and D. Donoho, "On denoising and best signal representation," IEEE Trans. on Information Theory, vol. 45 , no. 7, pp. 2225-2238, 1999.

[29] N. Ouarti and G. Peyré, "Best basis denoising with non-stationary wavelet packets," in 2009 16th IEEE International Conference on Image Processing (ICIP). IEEE, 2009, pp. 3825-3828.

[30] M. Elad, "Why simple shrinkage is still relevant for redundant representations?" IEEE Trans. on Information Theory, vol. 52, no. 12, pp. 5559-5569, 2006.

[31] J.-L. Starck, J. Fadili, and F. Murtagh, "The undecimated wavelet decomposition and its reconstruction," IEEE Trans. on Image Processing, vol. 16, no. 2, pp. 297-309, 2007.

[32] G. H. Golub and C. F. Van Loan, Matrix Computations. JHU press, 2012, vol. 3.

[33] A. Hyvärinen, P. O. Hoyer, and E. Oja, "Sparse code shrinkage: Denoising by nonlinear maximum likelihood estimation," in Advances in Neural Information Processing Systems, 1999, pp. 473-479.

[34] P. Moulin and J. Liu, "Analysis of multiresolution image denoising schemes using generalized-gaussian priors," in Proceedings of the IEEESP International Symposium on Time-Frequency and Time-Scale Analysis. IEEE, 1998, pp. 633-636.

[35] F. Xiao and Y. Zhang, "A comparative study on thresholding methods in wavelet-based image denoising," Procedia Engineering, vol. 15, pp. 3998-4003, 2011.

[36] Y. Hel-Or and D. Shaked, "Slicing the transform - a discriminative approach for Wavelet denoising," Hewlett-Packard Labs, Tech. Rep. HPL-2006-103R1, 2006.

[37] S. Dasgupta and A. Gupta, "An elementary proof of a theorem of Johnson and Lindenstrauss," Random Structures \& Algorithms, vol. 22, no. 1, pp. 60-65, 2003. 